\title{
Imaging Manifestations of Accessory Cavitated Uterine Mass-A Rare Mullerian Anomaly
}

\author{
Tharani Putta10 Reetu John² Betty Simon² Kirthi Sathyakumar2® Anuradha Chandramohan ${ }^{2}$ \\ Anu Eapen ${ }^{2}$ \\ ${ }^{1}$ Department of Radiology, AIG Hospitals, Hyderabad, Telangana, \\ India \\ 2 Department of Radiology, CMC, Vellore, Tamil Nadu, India \\ Address for correspondence Dr. Tharani Putta, MD, Department of \\ Radiology, AIG Hospitals, Gachibowli, Hyderabad, Telangana 500032, \\ India (e-mail: tharaniputta@gmail.com).
}

Indian J Radiol Imaging 2021;31:545-550.

\begin{abstract}
Keywords

- uterine cavity

- ACUM

- cavitated uterine mass

- cystic adenomyoma

- noncommunicating uterine horn

Context Accessory cavitated uterine mass (ACUM) is an uncommon and underrecognized entity with distinct imaging characteristics and causing significant patient distress. Differentiating it from its other clinical and radiological differentials is therefore extremely important and prevents delay in surgical management which is the treatment of choice.

Aims The aim of the study is to describe the MRI appearance of the surgically and pathologically proven ACUM cases from our institution in the last 2 years.

Settings and Design This is a retrospective study in a tertiary care hospital in South India.

Methods and Material We reviewed the clinical presentations and imaging findings of seven surgically proven cases of ACUM qualifying the proposed diagnostic criteria. Results All patients presented with chronic pelvic pain, dysmenorrhea, and prolonged post-menstrual pain. MRI in all seven cases showed an intramural, noncommunicating, and cavitating lesion near the uterine cornua with internal contents similar to that of endometrioma. Although the cavity was lined by endometrium in all the cases (proven in pathology), it was well appreciable on MRI in only five cases. The rest of the uterine myometrium and main endometrial cavity were normal with no features of adenomyosis.

Conclusion $\mathrm{MRI}$ is a reliable diagnostic tool for accurate diagnosis of ACUM, and more importantly, in distinguishing it from other causes of chronic pelvic pain like adenomyosis and endometriosis and other imaging differentials like adenomyoma, noncommunicating uterine horn, and degenerating leiomyoma.
\end{abstract}

\section{Introduction}

Accessory cavitated uterine mass (ACUM) is a relatively under-recognised entity characterized by the presence of a noncommunicating accessory cavity lined by endometrium and surrounded by uterine smooth muscle. ${ }^{1}$ It is typically located within the uterus close to the round ligament and has a uterus-like structural arrangement. ${ }^{2}$ The main endometrial cavity, fallopian tubes, and ovaries are normal. This entity has been previously reported in the literature as juvenile
DOI https://doi.org/ 10.1055/s-0041-1735504. ISSN 0971-3026.

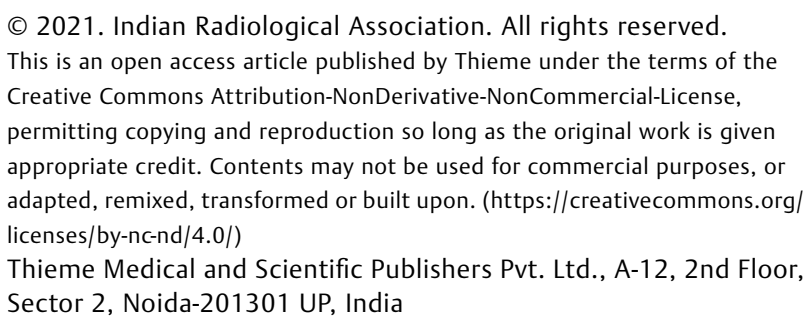

(C) 2021. Indian Radiological Association. All rights reserved. This is an open access article published by Thieme under the terms of the Creative Commons Attribution-NonDerivative-NonCommercial-License, permitting copying and reproduction so long as the original work is given appropriate credit. Contents may not be used for commercial purposes, or adapted, remixed, transformed or built upon. (https://creativecommons.org/ licenses/by-nc-nd/4.0/) Thieme Medical and Scientific Publishers Pvt. Ltd., A-12, 2nd Floor, Sector 2, Noida-201301 UP, India 
cystic adenomyoma, isolated cystic adenomyoma, and cavitated uterus-like mass within the myometrium, which are all now thought to represent ACUM. Although there are a few theories ${ }^{3}$ regarding the development of ACUM, most authors consider ACUM as a congenital Mullerian anomaly which is different from other Mullerian anomalies because of the otherwise normal endometrial cavity. It is hypothesized that this entity could be attributed to the duplication and persistence of a segment of Mullerian duct at the level of round ligament attachment, possibly due to Gubernaculum dysfunction. ${ }^{1,4}$

In this retrospective review, we highlighted the distinct clinical presentation and imaging findings of ACUM that will help in differentiating this condition from its other more common clinical differentials like adenomyosis and endometriosis, and other imaging differentials like true cavitating adenomyoma, noncommunicating functional uterine horn, and degenerating leiomyoma.

\section{Subjects and Methods}

In our retrospective study, we reviewed seven cases whose imaging showed isolated noncommunicating cavitated uterine lesion that was eventually proven to be ACUM based on the diagnostic criteria proposed by Acién et al. ${ }^{1}$ We also described the clinical presentation, MRI findings of these patients in detail, and reviewed the relevant literature.

\section{Diagnostic Criteria for ACUM}

1. An isolated accessory cavitated mass.

2. Normal uterus (endometrial cavity), tubes, and ovaries.

3. Surgical case with excised mass and pathological examination.

4. Accessory cavity lined by endometrial epithelium with glands and stroma.

5. Chocolate brown-colored fluid content.

6. No adenomyosis (if the uterus is removed) but there could be small foci of adenomyosis in the myometrium adjacent to the accessory cavity.

\section{Results}

Five out of our seven patients were under 30 years of age and unmarried. The remaining two patients who were over 30 years of age had two children each, one of whom delivered through caesarean sections both the times during which the accessory cavity was not recognized. One patient underwent emergency laparoscopic ovarian cystectomy for torsion, during which the bulge on uterus (from the ACUM) was presumed to be a pseudo-broad ligament fibroid and was left intact; surgical specimen histopathology subsequently confirmed ovarian serous cystadenoma. All our patients presented with chronic pelvic pain and severe dysmenorrhea starting a few years after menarche and five of them complained of prolonged post-menstrual pain lasting for up to 20 days. It is interesting to note that one of the patients reported a symptom-free interval during pregnancy and the post-partum period. All the patients received a combination of pain killers, OCPs and Dienogest (synthetic oral progestin) for varied clinical diagnosis (fibroid, endometriosis, rudimentary uterine horn, and ureteric colic). Six of them had an ultrasound scan prior to MRI and were told to have a fibroid. Review of the ultrasound images showed a cavity with endometrioma-like contents in five of the six patients (-Figs 1 and 2). Five patients underwent pelvic MRI (one of them in a scan centre elsewhere) and two of them underwent CECT abdomen and pelvis following which limited pelvic MRI was done by the radiologist. None of the patients underwent HSG or hysterosalpingogram ( - Table $\mathbf{1}$ ).

The MRI of all patients showed a cavitated lesion in the uterus (-Figs. 1-3) just anterior to the uterine cornua and corresponding to the round ligament attachment, three on the right side and four on the left with the size ranging from 3 to $4.3 \mathrm{~cm}$. All the lesions showed T1 hyperintense content with a T2 shading sign. The lesion wall was formed by T2 hypointense myometrium with thickness ranging from 8 to $11 \mathrm{~mm}$. Endometrial lining in the accessory cavity could be seen in five out of the seven patients and could not be distinctly seen in two of the patients. Rest of the uterus
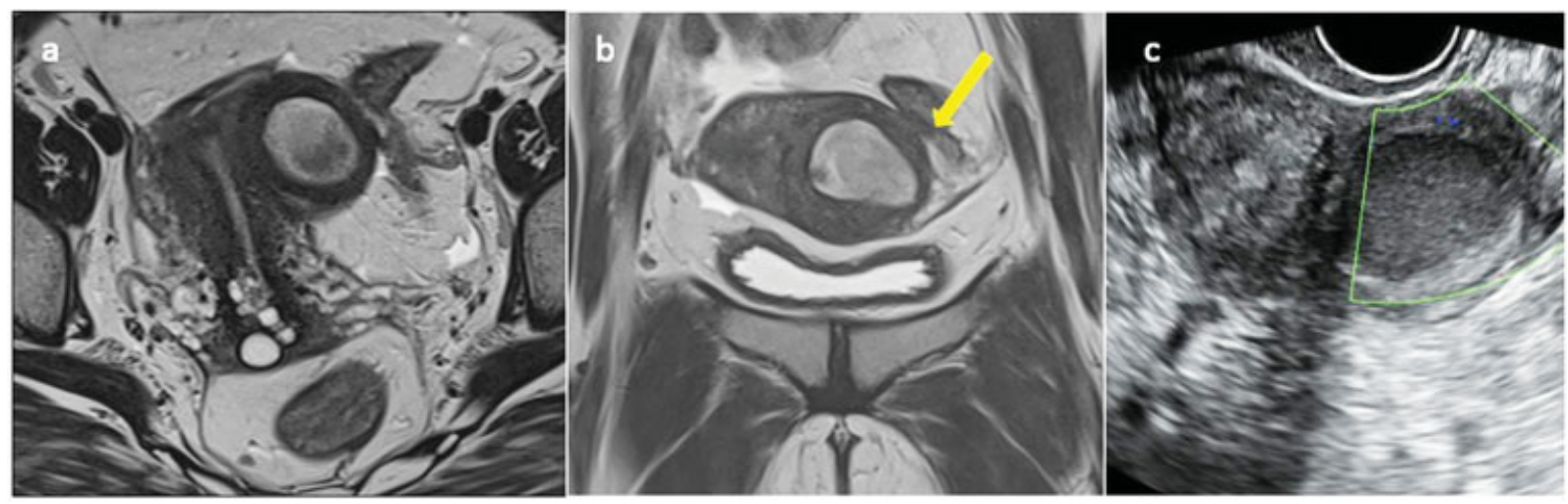

Fig. 1 T2 HR axial (a) and coronal (b) MR images show a thick-walled cavity in the left juxta-cornual location of the uterus lined by thin hyperintense endometrium and showing intermediate signal intensity fluid within. The fallopian tube attachment is seen posterior to the cavity (arrow in b). Representative transvaginal ultrasound image in the transverse plane (c) shows a cavity with ground-glass contents surrounded by hyperechoic endometrial lining and a thicker hypoechoic wall around it. 


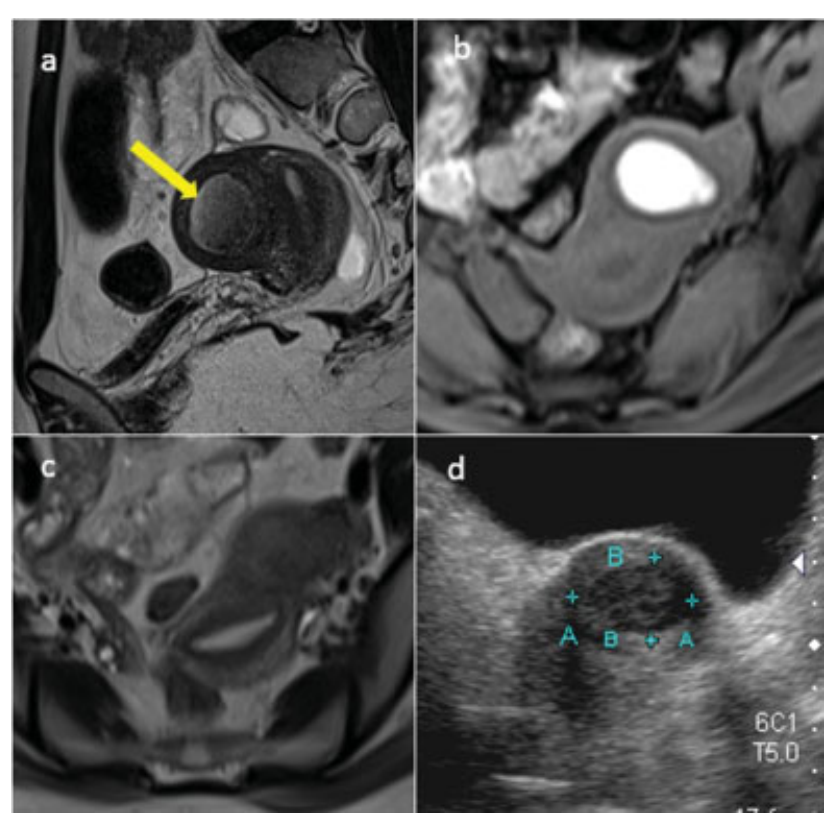

Fig. 2 T2W HR axial MRI (a) and T1W axial MRI (b) showing a thickwalled cavitated lesion anterior to the left uterine cornua, lined by T2 hyperintense endometrium (arrow in a). The contents of the cavity are of intermediate signal intensity with a "shading" sign on T2W image (a) and hyperintense on T1W image (b). Bilateral normal uterine cornua are seen separately in image (c). Representative ultrasound image (d) showing a hypoechoic lesion in the left anterior aspect of uterus with central cavitation and ground-glass content within.

including the endometrial cavity was normal in all patients with two well-seen uterine cornua, with the exception of a small intramural fibroid in one patient. Bilateral ovaries were normal except for one patient with a 4-cm hemorrhagic cyst in one of the ovaries (which spontaneously resolved by the time of surgery) and another patient with a 4-cm corpus luteal cyst. A preoperative diagnosis of ACUM was suggested based on the MRI findings in four of the cases while in the other three cases, the differentials of cystic adenomyoma and functional noncommunicating rudimentary uterine horn were raised at the time of prospective MRI reporting.

All patients underwent laparoscopic excision of the lesions. Intraoperatively, the lesions were beneath the round ligament attachment and ipsilateral fallopian tubes were seen along the posterior aspect of the lesion. The cavities had altered blood within and there was no communication with the main endometrial cavity. One of the patients was found to have intraoperative ipsilateral pelvic endometriosis.

The pathological examination of the excision specimens showed a myometrial wall with endometrial lining. Three of the patients also showed foci of adenomyosis within the wall of the cavity, although MRI did not show any evidence of endometrial tissue within the myometrium around the main cavity or the ACUM.

We were able to follow-up four patients ( 2 months to 2 years after surgery) who did not have any gynecological complaints. Two patients were lost to follow-up. One of the most recently operated patients had not yet come for first follow-up.

\section{Discussion}

Although ACUM was named so and clearly defined as a distinct clinical entity in 2010 by Acién et al. ${ }^{1}$ awareness about this condition among radiologists and gynecologists is still not widespread. To date, we have found 58 cases described in the literature satisfying the diagnostic criteria for ACUM. Of these, 24 cases were reported prior to the index article under various terminologies, and 34 cases were reported after 2010 (including four cases in the index article). The largest case series

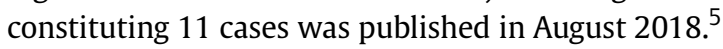

It is now clear that juvenile or isolated cystic adenomyomas and ACUM represent the same pathology. The typical patient was thought to be nulliparous under the age of 30 years, although it is now fairly clear that some of these women present later in life ${ }^{6}$ and some after previous childbirths. These women present with chronic pelvic pain and severe dysmenorrhea lasting for a few weeks after menstruation that is explained by the increased intracystic pressure following menstruation.

Ultrasound shows an intramural lesion which may be mistaken for a degenerating intramural or pseudo-broad ligament fibroid; however, the presence of a central single cavity with a ground-glass appearance of internal contents should raise the suspicion for ACUM.

MRI can help in localizing the lesion which is almost always seen at the uterine horn/cornua, beneath the round ligament and just anterior to the ipsilateral fallopian tube attachment. A few authors have reported the slightly more anterior location of the cavitated mass in the myometrium ${ }^{7}$ and more posteriorly in the myometrium close to the broad ligament. ${ }^{8}$ The contents of the cavity appear hyperintense on $\mathrm{T} 1 \mathrm{~W}$ or $\mathrm{T} 1 \mathrm{~W}$ fat-saturated images and intermediate signal to hypointense on T2W MRI, sometimes with a T2 shading effect. ${ }^{5,6}$ The cavity is lined by a thin T2 mildly high-signal-intensity endometrial lining (similar to that of the endometrial lining in the main endometrial cavity) which may or may not be well seen on T2-weighted MRI. The wall of the cavity appears T2 hypointense and is made up of organized uterine smooth muscle tissue. ${ }^{5}$ Although the wall of the accessory cavity can show foci of adenomyosis, there should not be any adenomyotic changes in the myometrium surrounding the main endometrial cavity. There may be an occasional rudimentary accessory fallopian tube attached to the mass, ${ }^{6}$ which is not necessarily seen on MRI. Bilateral ovaries and fallopian tubes are otherwise normal. There are no reported cases of associated renal or other congenital/developmental anomalies.

HSG may have an indirect role in the diagnosis of ACUM by revealing the morphology of native endometrial cavity. For example, a unicornuate morphology of the endometrial cavity may suggest the possibility of a noncommunicating rudimentary horn and ACUM is more likely to have a normal endometrial cavity with two distinct cornua on an anteroposterior spot image of HSG. But the literature available on this is limited. On the other hand, MRI helps in assessing the uterine wall in its entirety and is by far the gold standard noninvasive diagnostic modality for various myometrial lesions. 


\begin{tabular}{|c|c|c|c|c|c|c|c|c|c|c|c|c|c|c|}
\hline $\begin{array}{l}\hat{y} \\
\tilde{u} \\
\tilde{u}\end{array}$ & ๑ & 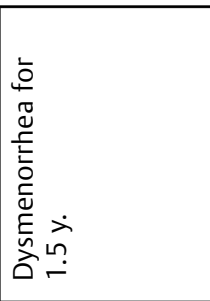 & 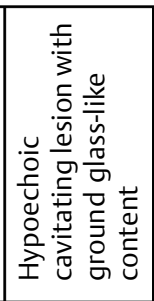 & $\mid$ & 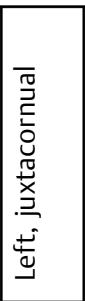 & 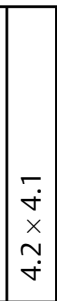 & 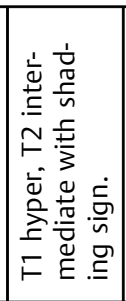 & a & 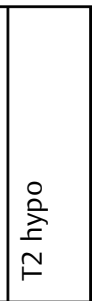 & $\mid \begin{array}{l}\bar{\Xi} \\
\stackrel{\varpi}{\sharp} \\
\overline{\bar{\Xi}} \\
3\end{array}$ & \begin{tabular}{|l}
$\overline{\widetilde{T}}$ \\
$\tilde{\Xi}$ \\
$\bar{z}$
\end{tabular} & \begin{tabular}{|l}
$\overline{\widetilde{T}}$ \\
$\stackrel{\underline{\Xi}}{0}$ \\
$\tilde{z}$
\end{tabular} & 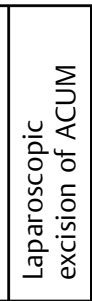 & 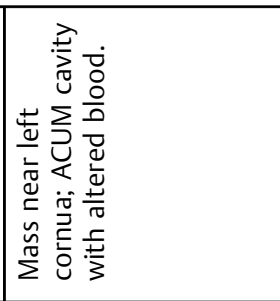 \\
\hline $\begin{array}{l}0 \\
\stackrel{\nu}{ } \\
\tilde{\Xi}\end{array}$ & $\underset{\sim}{ }$ & 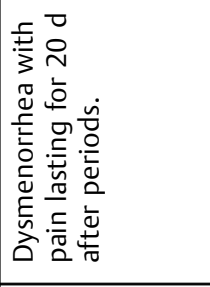 & 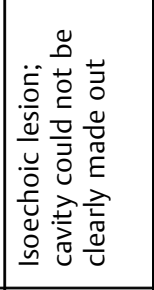 & \begin{tabular}{|l|}
$\mid$ \\
$\frac{1}{+}$ \\
\end{tabular} & 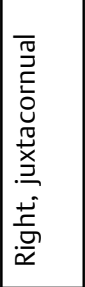 & $\mid \begin{array}{l}m \\
x \\
m \\
m \\
m\end{array}$ & 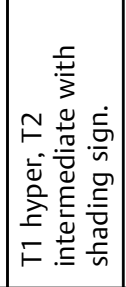 & 은 & 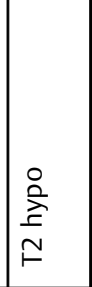 & 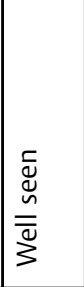 & \begin{tabular}{|l}
$\overline{\widetilde{T}}$ \\
$\stackrel{\bar{E}}{0}$ \\
$\bar{z}$
\end{tabular} & $\mid \begin{array}{l}\overline{\widetilde{O}} \\
\tilde{E} \\
\bar{z}\end{array}$ & 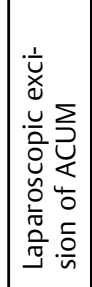 & 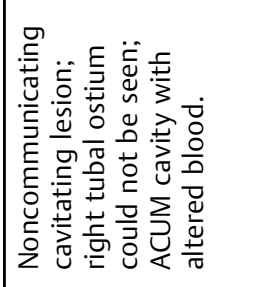 \\
\hline $\begin{array}{l}\text { n } \\
\tilde{\Delta} \\
\tilde{u}\end{array}$ & $\approx$ & 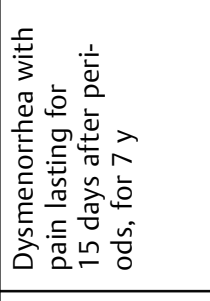 & 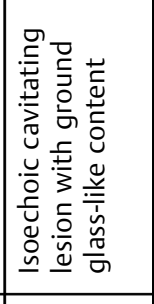 & \begin{tabular}{|} 
\\
+ \\
+ \\
\end{tabular} & 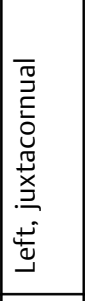 & $\begin{array}{c}\stackrel{n}{p} \\
\tilde{m} \\
\times \\
m \\
\dot{r} \\
\end{array}$ & 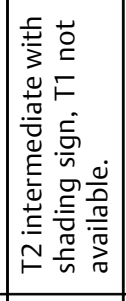 & $=$ & 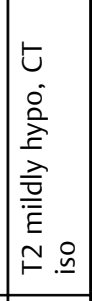 & $\mid \begin{array}{l}\bar{\Xi} \\
\stackrel{\Xi}{\tilde{n}} \\
\overline{\bar{\omega}} \\
3\end{array}$ & 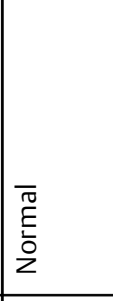 & \begin{tabular}{|l}
$\overline{\widetilde{T}}$ \\
$\stackrel{\underline{\Xi}}{0}$ \\
$z$
\end{tabular} & 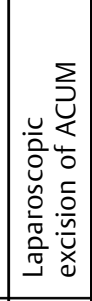 & 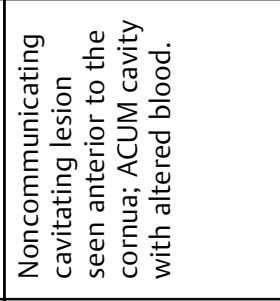 \\
\hline 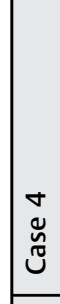 & $m$ & 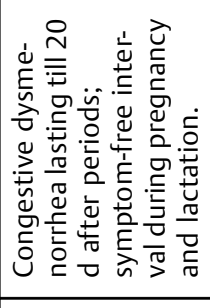 & « & \begin{tabular}{|}
1 \\
$\frac{1}{+}$ \\
\end{tabular} & 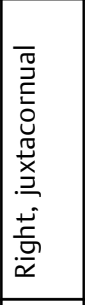 & \begin{tabular}{|l}
$\infty$ \\
$\dot{\sim}$ \\
$\dot{x}$ \\
$\dot{v}$ \\
$\dot{m}$ \\
$\dot{m}$
\end{tabular} & 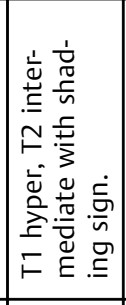 & a & 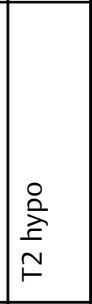 & 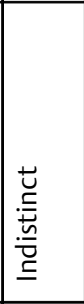 & \begin{tabular}{|l}
$\overline{\widetilde{N}}$ \\
$\tilde{\underline{\Xi}}$ \\
$\bar{z}$
\end{tabular} & \begin{tabular}{|l}
$\overline{\widetilde{T}}$ \\
$\tilde{E}$ \\
$\bar{z}$
\end{tabular} & 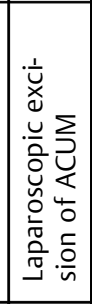 & 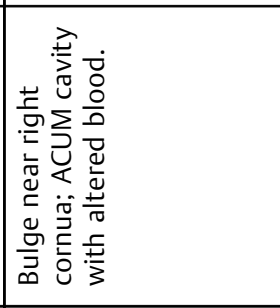 \\
\hline $\begin{array}{l}m \\
\tilde{u} \\
\tilde{u} \\
\tilde{u}\end{array}$ & $\bar{\sim}$ & 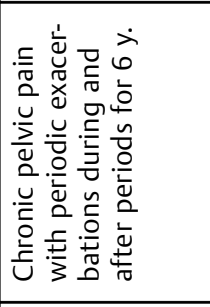 & 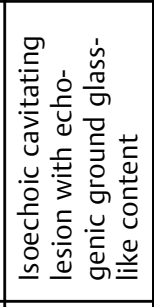 & $\begin{array}{r} \\
+ \\
+ \\
\end{array}$ & 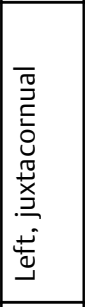 & \begin{tabular}{|c}
$n$ \\
$n$ \\
$\tilde{n}$ \\
$\times$ \\
$\dot{\sigma}$ \\
\end{tabular} & 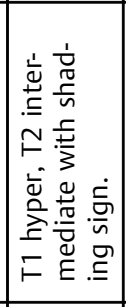 & $=$ & 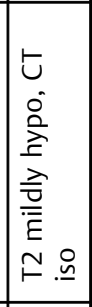 & 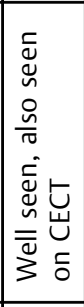 & \begin{tabular}{|l}
$\overline{\widetilde{\pi}}$ \\
$\tilde{\xi}$ \\
$\tilde{z}$ \\
\end{tabular} & 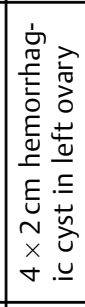 & 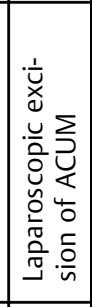 & 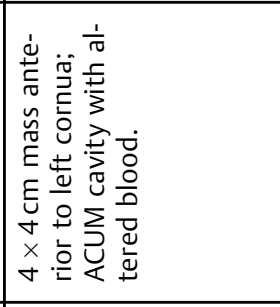 \\
\hline $\begin{array}{l}N \\
\tilde{u} \\
\tilde{u}\end{array}$ & $\stackrel{\sim}{\sim}$ & 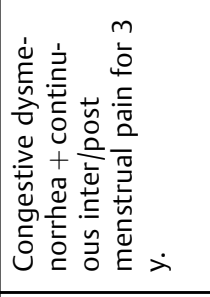 & 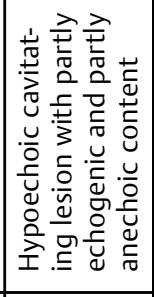 & \begin{tabular}{|c} 
\\
$\frac{1}{+}$
\end{tabular} & 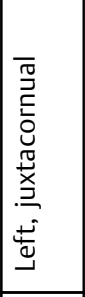 & $\begin{array}{c}m \\
x \\
m \\
m \\
m\end{array}$ & 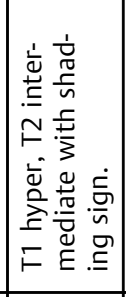 & $\infty$ & 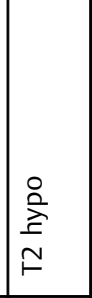 & $\begin{array}{l}\bar{\Xi} \\
\stackrel{\Xi}{ } \\
\overline{\bar{\Xi}} \\
3\end{array}$ & $\mid \begin{array}{l}\overline{\widetilde{T}} \\
\tilde{\Xi} \\
\dot{z}\end{array}$ & \begin{tabular}{|l}
$\overline{\widetilde{\pi}}$ \\
$\tilde{\xi}$ \\
$\dot{z}$
\end{tabular} & 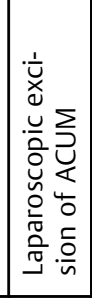 & 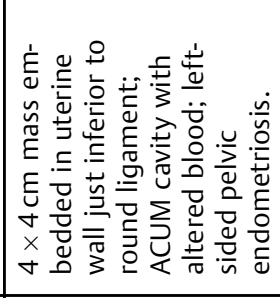 \\
\hline $\begin{array}{l}\bar{\Xi} \\
\stackrel{\tilde{U}}{0}\end{array}$ & $=$ & 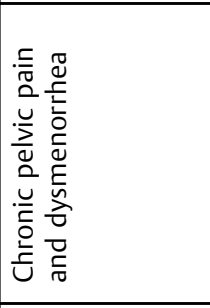 & 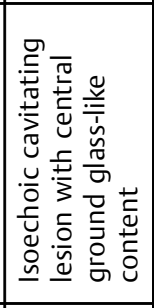 & $\frac{1}{+}$ & 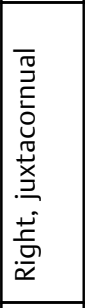 & $\begin{array}{l}\stackrel{m}{\tilde{N}} \\
\times \\
m \\
\end{array}$ & 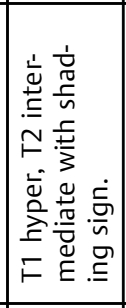 & $\circ$ & 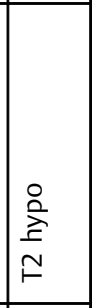 & 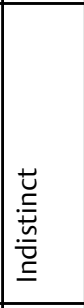 & 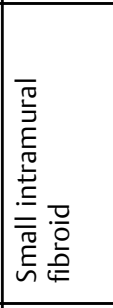 & 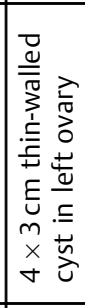 & 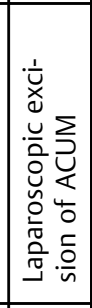 & 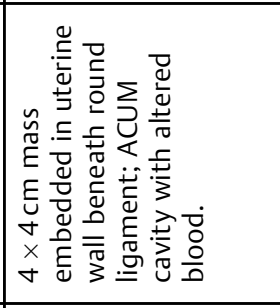 \\
\hline & 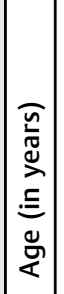 & 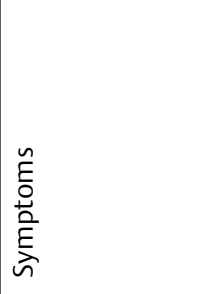 & 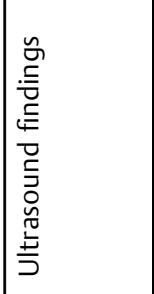 & $\begin{array}{l} \\
\frac{\mathrm{U}}{\mathrm{u}} \\
\frac{\mathrm{q}}{\Sigma}\end{array}$ & 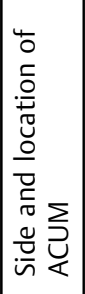 & 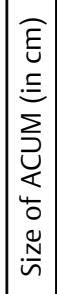 & 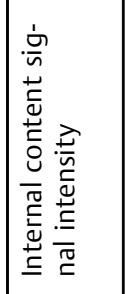 & 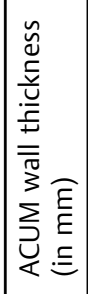 & 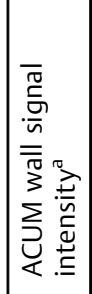 & 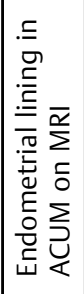 & 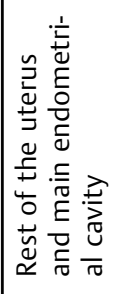 & 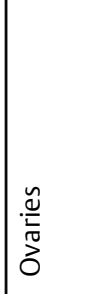 & 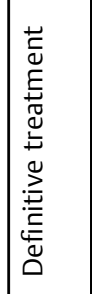 & 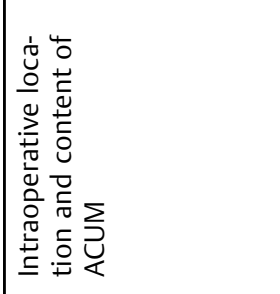 \\
\hline
\end{tabular}




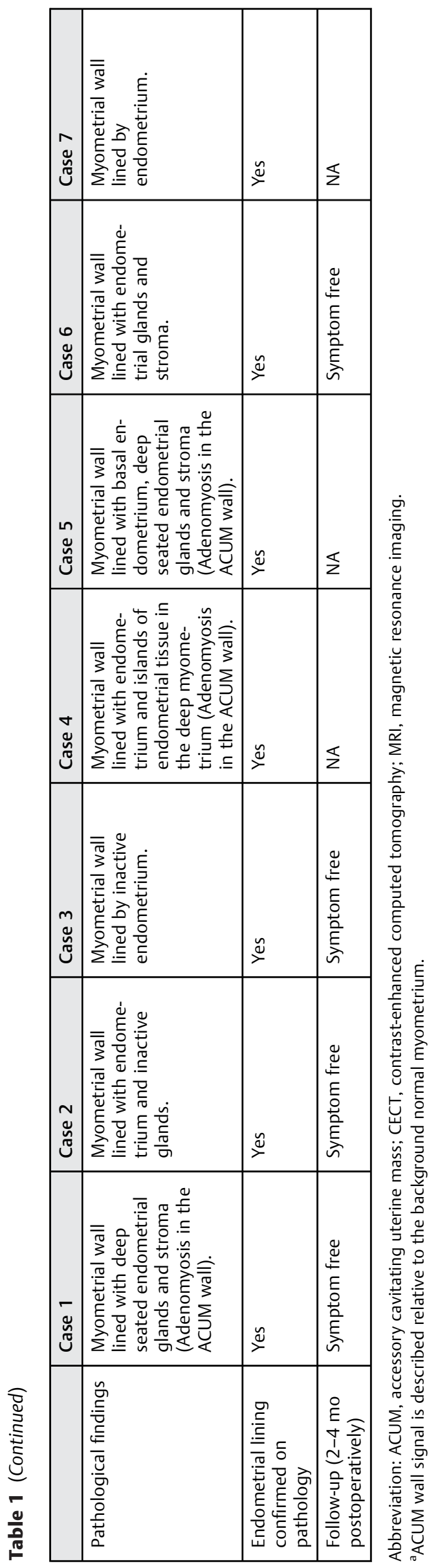

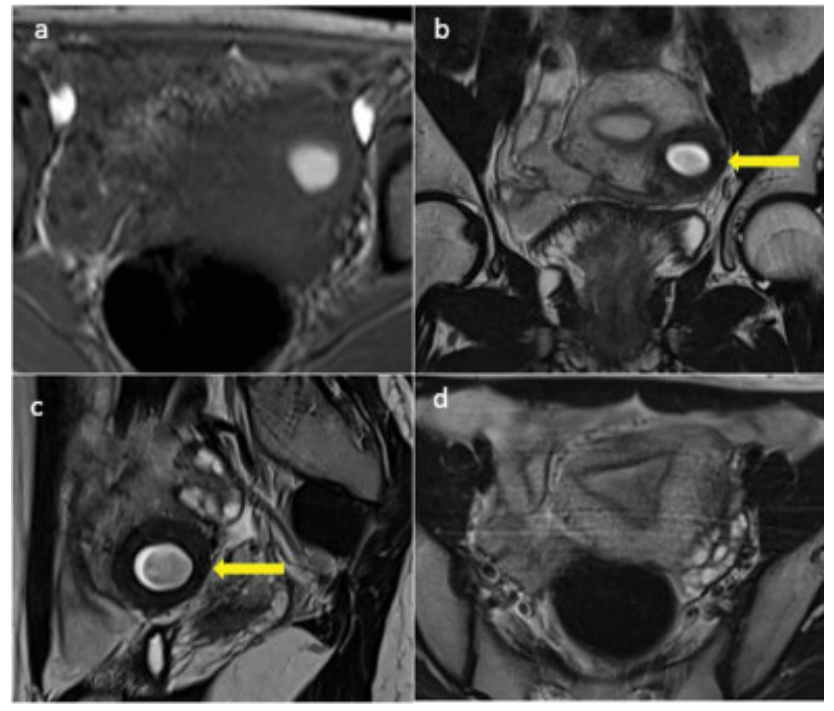

Fig. 3 Axial T1W MRI (a), T2 HR coronal (b), sagittal (c), and axial (d) MR images showing a cavitated lesion in the left side of uterus with its wall formed by $\mathrm{T} 2$ hypointense myometrium (yellow arrows). Internal contents are hyperintense on T1W image (a) and intermediate signal intensity with "shading" on T2W images (b and c). There is no communication between this lesion and the normal endometrial cavity. Bilateral normal cornua are shown in (d) excluding the possibility of a noncommunicating rudimentary functional uterine horn.

Clinical and imaging differentials for cavitated uterine lesion include true cavitated adenomyoma, functional noncommunicating rudimentary uterine horn, and cystic degeneration of a fibroid.

\section{ACUM versus True Adenomyoma}

- True adenomyomas can present in a similar manner clinically but in older women with severe adenomyosis; these lesions have ill-defined margins and do not have a topographic preference within the uterus, except for being close to the junctional zone. In adenomyoma, the junctional zone around the main uterine cavity is also thickened $(>12 \mathrm{~mm}$ ) with or without small $\mathrm{T} 1$ hyperintense hemorrhagic foci within.

- Cystic changes in adenomyoma are more often seen as multiple small cystic foci, although rarely a single cavity may be seen. ${ }^{9,10}$ In such cases, the lack of true endometrial lining and uterus-like structural and histological organization help differentiate it from ACUM. ${ }^{6}$

- Myometrium around the ACUM can show adenomyosis (as seen in the histopathology of three of our cases) due to the increased intracystic pressure, ${ }^{6}$ while the rest of the uterus does not show evidence of adenomyosis.

\section{ACUM versus Functional Noncommunicating Rudimentary Uterine Horn}

- ACUM can be differentiated from functional noncommunicating rudimentary uterine horn by demonstrating the normal native endometrial cavity with two normal cornua on MRI, although technically ACUM can rarely co- 
exist with other Mullerian anomalies. ${ }^{11}$ In the case of functional noncommunicating uterine horn, MRI shows the unicornuate morphology of the uterus on the side opposite to the rudimentary horn.

- MRI obviates the need for HSG or hysteroscopy which is recommended by a few authors. ${ }^{4,12}$

- Rudimentary functional uterine horn and ACUM have similar structural and histological organizations, and therefore, a pathologist cannot distinguish between these two entities when examining the surgical specimen, unless provided with the imaging findings of normal background uterine cavity or alternatively, a hysterectomy specimen is available.

\section{ACUM versus Leiomyoma with Cystic Degeneration}

- Uncommonly, leiomyomas may demonstrate a single, central cystic degeneration but the contents are rarely hemorrhagic. The lack of topographic preference within the uterus, lack of cyclical pain, and possible presence of multiple lesions help in differentiating this uncommon manifestation of leiomyoma from ACUM. ${ }^{5}$

Definitive management is complete surgical excision of the mass, preferably using laparoscopy. ACUM has well-defined margins and complete enucleation is possible without much myometrial damage. Whenever possible, anterior incision over the uterine mass is preferred over other surgical approaches. ${ }^{5}$

One of our patients was diagnosed to have superficial pelvic endometriosis intraoperatively. Although this finding was not initially described with reference to ACUM, there are at least two other ACUM cases described in the literature showing superficial pelvic endometriosis without evidence of adenomyosis. ${ }^{5}$

Uncommon manifestations of ACUM reported in the literature include:

More than one accessory cavity in the same patient, ${ }^{6}$ which is an exception for the first diagnostic criterion; ACUM can co-exist with other Mullerian anomalies, ${ }^{11}$ which is an exception for the second diagnostic criterion; One published case of twin pregnancy in the ACUM cavity. ${ }^{13}$

Similar extra-uterine uterus-like masses (ULMs) have been reported in the literature in vagina, ovaries, broad ligament, uterosacral ligament, pelvic sidewall, small bowel mesentery, sigmoid mesocolon, along the caecum and colonic wall, inguinal region, and conus medullaris. ${ }^{3}$

\section{Conclusion}

ACUM is now widely accepted as an uncommon Mullerian abnormality causing significant patient distress. Although an uncommon condition, its characteristic MRI findings allow accurate diagnosis to be made by radiologists. Early surgical excision is the treatment of choice and provides complete symptomatic relief.

\author{
Funding \\ None. \\ Conflict of Interest \\ None declared.
}

\section{References}

1 Acién P, Acién M, Fernández F, José Mayol M, Aranda I. The cavitated accessory uterine mass: a Müllerian anomaly in women with an otherwise normal uterus. Obstet Gynecol 2010;116(05): 1101-1109

2 Jain N, Verma R. Imaging diagnosis of accessory and cavitated uterine mass, a rare Mullerian anomaly. Indian J Radiol Imaging 2014;24(02):178-181

3 Na KY, Kim GY, Won KY, et al. Extrapelvic uterus-like masses presenting as colonic submucosal tumor: a case study and review of literature. Korean J Pathol 2013;47(02):177-181

4 Acién P, Sánchez del Campo F, Mayol M-J, Acién M. The female gubernaculum: role in the embryology and development of the genital tract and in the possible genesis of malformations. Eur J Obstet Gynecol Reprod Biol 2011;159(02):426-432

5 Peyron N, Jacquemier E, Charlot M, et al. Accessory cavitated uterine mass: MRI features and surgical correlations of a rare but under-recognised entity. Eur Radiol 2019;29(03): $1144-1152$

6 Acién P, Bataller A, Fernández F, Acién MI, Rodríguez JM, Mayol MJ. New cases of accessory and cavitated uterine masses (ACUM): a significant cause of severe dysmenorrhea and recurrent pelvic pain in young women. Hum Reprod 2012;27(03): 683-694

7 Kriplani A, Mahey R, Agarwal N, Bhatla N, Yadav R, Singh MK. Laparoscopic management of juvenile cystic adenomyoma: four cases. J Minim Invasive Gynecol 2011;18(03):343-348

8 Chun SS, Hong DG, Seong WJ, Choi MH, Lee TH. Juvenile cystic adenomyoma in a 19-year-old woman: a case report with a proposal for new diagnostic criteria. J Laparoendosc Adv Surg Tech A 2011;21(08):771-774

9 Bazot M, Daraï E Role of transvaginal sonography and magnetic resonance imaging in the diagnosis of uterine adenomyosis. Fertil Steril 2018;109(03):389-397

10 Tamai K, Togashi K, Ito T, Morisawa N, Fujiwara T, Koyama T. MR imaging findings of adenomyosis: correlation with histopathologic features and diagnostic pitfalls. Radiographics 2005;25(01):21-40

11 Persson J, Bossmar T, Teleman P. Robot-assisted laparoscopic surgery for a rudimentary uterine horn with two non-communicating cavities. J Robot Surg 2010;4(02):137-140

12 Acién P, Acién MI. The history of female genital tract malformation classifications and proposal of an updated system. Hum Reprod Update 2011;17(05):693-705

13 Alkhateeb HM, Yaseen EM. Twin pregnancy in an accessory cavitated non-communicating uterus. Int J Surg Case Rep 2015; $10: 45-48$ 